\title{
NeuroRegulation
}

\section{Neurofeedback Requires Better Evidence of Efficacy Before It Should Be Considered a Legitimate Treatment for ADHD: What is the Evidence for this Claim?}

\author{
H. Edmund Pigott, Ph.D. * \& Rex Cannon, Ph.D. \\ Brain Treatment Centers of South Florida
}

\begin{abstract}
*Address correspondence to: H. Edmund Pigott, Ph.D., Brain Treatment Centers of South Florida, $1101 \mathrm{~N}$ Congress Ave, Suite 202, Boynton Beach, Florida, 33426. Email: pathware@erols.com

Copyright: () 2014 Pigott and Cannon. This is an Open Access article distributed under the terms of the Creative Commons Attribution License (CC-BY).
\end{abstract}

Russell Barkley is the most prominent and longstanding critic of neurofeedback (NFB) treatment for ADHD (Barkley, 1992). In a 2005 review article coauthored with Sandra Loo, Loo/Barkley state that for NFB to be considered a "legitimate treatment" it must not only be found effective, but it also must be demonstrated in "studies that are scientifically rigorous" that:

- $\quad$ "Changing the EEG is the mechanism of change in ADHD symptoms;"

- The treatment effects must also "generalize to non-treatment settings" and "persist over time;" and furthermore,

- $\quad$ "Even with such demonstrations, it must also be shown that treatment is cost effective in managing the symptoms of ADHD relative to the prevailing empirically supported approaches" (Loo \& Barkley, 2005).

What is Good for the Goose is Good for the Gander

Logically, if we accept Loo/Barkley's evidentiary standards for NFB, the same standards should be applied even-handedly to all psychological and pharmaceutical treatments for ADHD. Call it the "What is Good for the Goose is Good for the Gander" rule; a rule that is critical to minimize bias when evaluating the evidence of different treatments. By applying this rule, there are simply no psychosocial OR pharmaceutical treatments for any behavioral health disorder that meet the Loo/Barkley evidentiary standards.

Dr. Barkley violates his own "treatment legitimacy" standards. Barkley and many others have been strong proponents of stimulant medications to treat ADHD since the mid-1970s; but despite billions of dollars spent in "scientifically rigorous" research efforts over the past 40+ years, we still do not know what are the mechanisms of change from stimulants that account for the observed improvements in randomized trials typically lasting only 6 to 8 weeks.

In the Physician Desk Reference, every psychoactive medication has a statement similar to "presumably works by" or "is thought to..." when describing an FDA-approved drug's hypothesized "mechanism of change;" yet Loo/Barkley fail to hold stimulants to the same evidentiary standard they assert is necessary for NFB to meet before it can be considered a legitimate treatment. Take methylphenidate for example, the most commonly prescribed drug for ADHD: 
The mode of therapeutic action in humans is not completely understood, but methylphenidate presumably activates the brain stem arousal system and cortex to produce its stimulant effect. Methylphenidate is thought to block the reuptake of norepinephrine and dopamine into the presynaptic neuron and increase the release of these monoamines into the extraneuronal space. There is neither specific evidence that clearly establishes the mechanism whereby Methylin produces its mental and behavioral effects in children, nor conclusive evidence regarding how these effects relate to the condition of the central nervous system. [emphases added; FDA, 2013]

It is also well known that the effects of stimulant medications do not "persist over time" when treatment is stopped. Barkley himself emphasizes this point on his website. Furthermore, there are now three publically-funded studies finding no evidence that the benefits of stimulants "persist over time" even from continued stimulant medication treatment (Molina et al., 2009; Smith et al., 2010; Riddle et al., 2013) with substantial evidence suggesting that such continued treatment for many becomes iatrogenic overtime (Pigott \& Cannon, 2014).

Regarding cost-effectiveness, medication-based treatment is expensive given the fact that in the attempts to sustain effectiveness, people have to take the medication(s) on an ongoing basis, and for many, at ever higher doses and/or with intermittent medication changes and new drug augmentation due to the habituation effects that commonly develop to the originally prescribed medication(s). This reality is seen in the Preschool Attention-Deficit/Hyperactivity Disorder Treatment (PATS) study (Riddle et al., 2013). By year 3, an antipsychotic had been added to $8.3 \%$ of the preschoolers' medication regimen (mean age: 7.4 years), and by year $6,12.9 \%$ were taking an antipsychotic (mean age: 10.4 years). This loss of efficacy in ADHD medications accounts for the majority of the dramatic increase in prescribing antipsychotics to children. In a 2012 article, Olfson et al. report that between 1993-1998 and 2005-2009, the rate of antipsychotics prescribed to children increased by over $750 \%$. Their analysis found that disruptive behavior disorders (primarily ADHD) were the most common diagnoses in children that were prescribed an antipsychotic accounting for $63 \%$ of such cases, and that in $54.1 \%$ of the outpatient visits, whenever an antipsychotic was prescribed there was also an ADHD medication prescribed to the same child.

The combination of open-ended treatment by medication(s), and the associated physician fees for overseeing the prescribing of these drugs, makes drug-centric treatment for ADHD very expensive with a poor cost-benefit return on investment as demonstrated by the MTA Cooperative study authors' own conclusion that they found no evidence to support the "longterm advantage of (continued) medication treatment beyond 2 years for the majority of children" (Molina et al., 2009); a conclusion identical to that found in the PATS and Australian studies (Riddle et al., 2013; Smith et al., 2010). The simple fact is that the available evidence from these large, taxpayer-funded studies indicates not only that the effects of stimulant medications do not "persist over time" after treatment is stopped, there is no evidence of a sustained benefit when stimulant drugs and new drug augmentations continue to be taken.

On the behavior therapy front, behaviorally-based parent training of the type developed by Barkley (1987) and classroom management strategies have not been subjected to rigorous controlled trials in which the specific aspects of the interventions were shown to be the mediating mechanisms of change, nor has it been shown that the observed changes generalized to other settings or persisted over time. In fact, we know that the effects of 
such behavioral strategies for ADHD do not generalize to other settings nor persist over time, as Barkley himself acknowledges on his website stating:

Psychological treatments, such as behavior modification in the classroom and parent training in child behavior management methods, have been shown to produce short-term benefits in these settings. However, the improvements which they render are often limited to those settings in which treatment is occurring and do not generalize to other settings that are not included in the management program. Moreover, recent studies suggest, as with the medications discussed above, that the gains obtained during treatment may not last once treatment has been terminated. Thus, it appears that treatments for ADHD must often be combined and must be maintained over long periods of time so as to sustain the initial treatment effects. In this regard, ADHD should be viewed like any other chronic medical condition that requires ongoing treatment for its effective management but whose treatments do not rid the individual of the disorder. [emphases added; Barkley website]

Neurofeedback Comes Closest to Meeting the Loo/Barkley Evidentiary Standards

The Loo/Barkley review article holds NFB to far higher evidentiary standards than are applied to the widely accepted treatments of stimulant medication and behavior therapy, thereby reflecting bias on their part and making it hard to take as credible their selective review of the NFB research. The irony here is that NFB comes far closer to meeting the Loo/Barkley evidentiary standards for the effective treatment of ADHD than either of these two widely accepted treatments. Consider for example:

- The very first NFB studies by Lubar and Shouse demonstrated that "changing the EEG is the mechanism of change in ADHD symptoms" (Lubar \& Shouse, 1976; Shouse \& Lubar, 1979). Using a scientifically rigorous within-subject reversal design with blinded raters, these researchers found that when ADHD boys were reinforced for increasing their sensory motor rhythm (SMR), their hyperactive and distractible/inattentive symptoms significantly decreased and these treatment gains were reversed when the boys were reinforced for decreasing SMR.

- In 1995, Lubar et al. demonstrated that ADHD children who learned to decrease their theta/beta ratios through NFB training showed improvement on multiple outcome measures while non-learners did not improve. Furthermore, four new studies correlated the extent of changes in subjects' EEG to ADHD symptom improvement, and those subjects who were most successful in learning to self-regulate their EEG had the greatest improvement in ADHD symptoms, thereby providing additional strong evidence that "changing the EEG is the mechanism of change in ADHD symptoms" resulting from NFB treatment. Finally, there are now seven studies demonstrating that NFB resulted in protocol-specified "changes in the EEG," and these improvements in EEG self-regulation persisted when reassessed at 6 months (2 studies) and 2 years ( 1 study) after treatment termination with associated sustained improvement in ADHD core symptoms (see Pigott \& Cannon, 2014). 
- In comparison to control group conditions, NFB has been shown to result in significant improvements in (a) parent-rated core symptoms of ADHD (15 studies); (b) teacher-rated core symptoms (12 studies); (c) computerized continuous performance tests of core symptoms (8 studies); (d) neuropsychological measures of response inhibition, reaction time, and concentration (4 studies); and (e) neurophysiologic measures of improvement relevant to ADHD including the QEEG Attention Index (1 study), Event-Related Potentials (P300) during continuous performance testing (1 study), and activation of regions in the brain related to attention and executive functioning when assessed using fMRI (1 study). These findings from numerous international research groups provide strong evidence that, unlike traditional behavior therapy, the gains from NFB treatment "generalize to non-treatment settings," and this generalization effect is the result of subjects learning how to self-regulate their EEG (see Pigott \& Cannon, 2014).

- In follow-up studies, NFB resulted in significant improvement in core ADHD symptoms that were sustained when reassessed at six months ( 5 studies) and 2 years ( 2 studies) after treatment termination, thereby providing strong evidence that, unlike stimulant medications and traditional behavior therapy, the gains from NFB treatment "persist over time" following treatment termination (see Pigott \& Cannon, 2014).

In contrast to Dr. Barkley's acknowledgement of the limited effectiveness for what are widely deemed as "legitimate treatments," those being open-ended medication(s) and behavioral management programs implemented across settings, such that "ADHD should be viewed like any other chronic medical condition that requires ongoing (combination) treatment(s) for its effective management but whose treatments do not rid the individual of the disorder," the Gani et al. (2008) study found at the two-year follow-up assessment of NFB's effectiveness: (a) "yet another significant reduction of number of (ADHD-related) problems and significant improvement in attention was observed," (b) "EEG self-regulation skills were preserved," (c) "half of the children no longer met ADHD criteria," and (d) only $22 \%$ were still taking medication for ADHD. These authors therefore concluded that,

"Neurofeedback appears to be an alternative or complement to traditional treatments. The stability of changes might be explained by normalizing of brain functions that are responsible for inhibitory control, impulsivity and hyperactivity."

\section{Negative Findings from a Recent Meta-Analysis}

Recently, the self-named European ADHD Guidelines Group conducted meta-analyses of randomized trials of six different non-pharmacological treatments using parent- and teachercompleted rating scales to compute each treatment's effect size (Sonuga-Barke et al., 2013a). This article found significant effect sizes for learning-based interventions using raters closest to the treatment setting (the so-called "most proximal assessment," parents for all home- and clinic-based interventions), but no significant effects using raters furthest from the treatment setting (the so-called "probably blinded assessment," typically teachers, except as the authors state, "If the intervention was implemented at school, teacher ratings were not considered probably blinded assessments"). The researchers concluded from these findings that "better evidence for efficacy from blinded assessments is required for behavioral interventions, neurofeedback, cognitive training (CT), and restricted elimination diets before they can be supported as treatments for core ADHD symptoms" [emphasis added].

While this group's approach was clever, their sweeping conclusions regarding NFB are not valid and instead demonstrate how meta-analyses are constrained by their methods and, 
therefore, their conclusions should be similarly constrained. In this regard, it is important to know that NFB's treatment effect size narrowly missed reaching significance $(p=.07)$ in the group's "probably blinded" meta-analysis, whereas CT $(p=0.34)$ and behavioral interventions $(p=0.92)$ did far worse, indicating that it would take only a slight shift in their methodology to find NFB efficacious, but not so for CT or behavioral interventions.

\section{Garbage In, Garbage Out}

There are several significant methodological errors in the group's meta-analyses that invalidate their conclusions. First, the article's methods section states, "Participants (ages 3 to 18 years) had a diagnosis of ADHD of any subtype (DSM-defined ADHD or ICD-defined hyperkinetic disorder ... OR met accepted criteria for clinical levels of symptoms on validated ADHD rating scales" [emphasis added]. The authors fail to acknowledge that such allegedly validated ADHD rating scales have only class-IV evidence for diagnosing ADHD according to the American Academy of Neurology, and in a recent large blinded multisite trial had exceptionally poor diagnostic sensitivity (range: 38-79\%) and specificity (range: 13-61\%) with an overall diagnostic accuracy similar to flipping a coin (range: $47-58 \%$; see Snyder et al., 2008). Rigorous methods would have informed readers which of their included studies relied on such "flip-a-coin" rating scales to diagnosis ADHD—versus the goldstandard of clinician diagnosis - and then analyzed these low-quality studies separately to assess if they differentially affected their findings. The inclusion of such studies in their metaanalyses, an error the authors then compounded by not assessing for the low-quality studies' differential impact, is a prime example of the "garbage-in-garbage-out" critique of methodologically flawed meta-analyses.

\section{Ignoring Neurofeedback's Use of Objective Outcomes}

Second, to analyze outcomes, the authors relied on parent and teacher-completed ADHD rating scales that are subject to significant rater bias with poor inter-rater agreement and exceedingly high levels of false-positive and false-negative findings when used to assess ADHD (Snyder et al., 2008). Due to not having both parent- and teacher-completed rating scales, the group excluded from its "probably blinded" meta-analysis randomized NFB studies using more objective measures of ADHD including:

- Computerized continuous performance testing of core ADHD symptoms (Heinrich et al. 2004; Bakhshayesh et al., 2011; Levesque et al., 2006);

- Neuropsychological measures of core ADHD symptoms including response inhibition, reaction time, and concentration (Holtmann et al., 2009; Bakhshayesh et al., 2011; Levesque et al., 2006); and

- Neurophysiological measures of improvement relevant to ADHD such as Event-Related Potentials (P300) during continuous performance testing (Heinrich et al., 2004) and activation of regions in the brain related to attention and executive functioning assessed using fMRI (Levesque et al., 2006).

Unlike other treatments in the group's meta-analyses, the use of such objective measures is common in NFB studies. In general, randomized NFB studies blindly administered these more objective measures, and they consistently demonstrate NFB's superiority over the control group conditions. Unfortunately, the guideline group not only excluded these measures from its "probably blinded" meta-analysis, thereby significantly reducing the number of NFB studies with positive findings included in it, but also failed to inform journal 
readers of the existence of these measures that are directly related to assessing the efficacy of ADHD treatments.

\section{Protocol Violation \#1}

Third, one of the trials in the group's "probably blinded" analysis was Steiner et al.'s preliminary study $(N=41)$ comparing NFB, CT and waitlist groups (Steiner et al., 2011). In this study, the NFB and CT treatments were provided at school in a separate room during "team time" twice per week for 4 months while the waitlist subjects remained with their class. Many of the teachers, therefore, were not "probably blinded" about which students left their classes twice each week for four months to be treated, though the teachers did not know if these departing students were receiving NFB or CT. Despite this fact, the researchers chose to compare NFB to the waitlist group using the teacher ratings for their "probably blinded" analysis versus comparing NFB to CT; a comparison that was both blinded and more rigorous.

In a Letter to the Editor, Arns and Strehl (2013) noted how Sonuga-Barke et al. violated their published protocol, which stated they selected control conditions "in the following order: sham/placebo, attention/ACTIVE CONTROL, treatment as usual, waiting list" [emphasis added; Sonuga-Barke et al., 2013a]. According to this protocol, the guideline group should have compared NFB to CT, not waitlist, with CT as the active control group condition. Arns and Strehl then recalculated Sonuga-Barke et al.'s meta-analysis, and this effort found NFB as having a significant effect size in the "probably blinded" analysis.

In their response letter, Sonuga-Barke et al. (2013b) claimed that they did not violate their protocol, stating that in Steiner et al. the "type of cognitive training was not considered a control condition but rather an optimized active ADHD treatment;" yet no such "optimized active" exclusion to their protocol is mentioned in the group's article. This rationale on Sonuga-Barke et al.'s part, therefore, appears more like a post hoc formulation to justify their protocol violation after it was discovered by Arns and Strehl than anything prespecified; otherwise, the "optimized active" exclusion would have been stated in their article.

\section{Lack of Blinding to Study Outcomes during Consensus Decision-Making Process}

Fourth, when selecting which control group to use for their "probably blinded" analysis, the group used a consensus decision-making approach with no attempt to keep group members blind to study outcomes prior to selection, a strategy that would have protected against members' biases for and against particular treatments. Similarly, the group used the same approach when deciding which measures to use for their "most proximal" and "probably blinded" meta-analyses. In response to our email inquiring about a second protocol violation we discovered, and specifically asking for evidence of the blinding of group members from knowledge of study outcomes in their decision-making processes, the group's leader, Joe Sergeant, wrote:

With respect to what you and your colleagues refer to as a "protocol violation", this matter was dealt with a year ago in the pre-publication correspondence with colleagues Arns and Strehl. Our response to them was: Our meta-analytic approach compared the treatment effects for most proximal and probably blinded measures. It placed an onus on us to make judgments about what was and was not the best probably blinded measure in each trial. Some times this decision was very 
straightforward at other times it was less so. In these cases we had to use ALL AVAILABLE INFORMATION within the papers to make a judgment about what constituted probable blinding. Including the term probably in its title explicitly acknowledged the judgment that was required by the reviewers in choosing this class of outcomes. In the case of the Steiner (2011) paper, after much examination of the available information and discussion we decided that the teacher-ratings were probably blinded while the parent ratings were not. First the treatment took place outside the regular classrooms during the school's "team time" (so that classroom instruction was not affected). Second, no teachers were involved in the delivery of the interventions. Third, the post intervention ratings were made by different teachers than the ones that made the pre-treatment ratings due to teacher changes following treatment in the new school. Fourth, in contrast, the researchers made no attempt to keep parents "blind" to the type of treatment. It seemed to us very likely that the parents knew which arm of the study the children were included in. Taking all these factors together our judgment was that the parent rating was probably not blinded while the teacher rating was. The statement in the paper "...if the intervention was implemented at school, teacher ratings were not considered probably blinded assessments..." referred essentially to classroom-based interventions involving teachers in their administration. However, the interventions in the Steiner et al. study, although they were delivered on school property, were not strictly speaking school-based intervention, in the sense of them being delivered by a teacher in the classroom [emphases added; Sergeant personal communication, 2014].

As is clear from Sergeant's response, group members were not blind to study outcomes when deciding either the group to use as NFB's control group comparator nor when assigning the parent and teacher ratings to their respective "most proximal" and "probably blinded" meta-analyses.

This lack of blinding to study outcomes is a significant methodological flaw, because the waitlist group in the Steiner et al. preliminary study had an unusually large effect size of .4 in the teacher ratings versus a negative effect size of .1 for CT (see Table 1). To our knowledge, a 4 effect size for a group of waitlisted ADHD children is unprecedented. It was unscientific for the group members to know these facts when deciding to use the teacher ratings of the waitlist group with its large effect size for their control group comparison in the "probably blinded' analysis versus the slight negative effect size for CT, and in so choosing violate their published protocol for guiding this decision.

Table 1: Effect Sizes in Steiner et al. Preliminary Study

\begin{tabular}{|l|c|c|c|}
\hline & Neurofeedback & Cognitive Training & Waitlist \\
\hline Aggregate of Parent Ratings & 1.1 & 0.5 & 0.0 \\
\hline Teacher Ratings & 0.2 & -0.1 & 0.4 \\
\hline
\end{tabular}




\section{Protocol Violation \#2}

Fifth, group members knew when deciding which measures to use for their "most proximal" and "probably blinded" meta-analyses that the parents rated the NFB group an exceptionally large effect size of 1.1 and a 0.0 effect size for the waitlist group, while the teachers rated NFB as having only a modest effect size of .2 and, as previously noted, an unprecedented .4 for the waitlist group -an effect size twice as large as the teachers rated NFB. With full access to this knowledge, the guideline group then chose the parents' ratings of NFB to include in their "most proximal" meta-analysis and use the teachers' ratings for their "probably blinded" meta-analysis despite their protocol specifically stating, "If the intervention was implemented at SCHOOL, teacher ratings WERE NOT considered probably blinded assessments" [emphasis added].

As part of the group's rationale for this protocol violation, Sergeant writes, "The post intervention ratings were made by different teachers than the ones that made the pretreatment ratings due to teacher changes following treatment in the new school ... in contrast, the researchers made no attempt to keep parents "blind" to the type of treatment."

Sergeant's claim would have a thin modicum of merit if the 4-month-long NFB and CT interventions CONCLUDED at the end of the school year and then, at the start of the new year, new teachers blind to which children had been removed during team time for NFB and CT were the ones who rated all subjects. The post-intervention ratings would then have been provided by teachers who were blind to which ADHD children were in the waitlist versus intervention groups. Just the opposite, though, is what occurred in the Steiner et al. study. In the methods section, Steiner et al. states, "The preintervention questionnaires were filled out at the time of enrollment at the end of the previous school year, and the postintervention questionnaires were filled out within 1 month after the intervention" [emphases added, Steiner et al., 2011]. Similar to the group's response to the Arns and Strehl letter claiming an "optimized active treatment" exclusion to their published protocol, hereto the group's rationale appears like another post hoc formulation attempting to justify their second violation to a clearly stated protocol.

\section{Lack of Scientific Rigor}

Finally, it was a significant abdication of scientific rigor that Sonuga-Barke et al. did not blind group members to study outcomes when deciding on (a) the assignment of control group conditions; and (b) which measures to use for the "most proximal/probably blinded" metaanalyses, as well as not ensuring the consistent adherence to their clearly stated protocols regarding these matters. This abdication of scientific rigor is especially the case here, since this was the whole point of Sonuga-Barke et al.'s 12 meta-analyses, which was to test the significance levels of the effect sizes for the "most proximal" and "probably blinded" assessments.

As Sergeant states, "Sometimes this decision was very straightforward at other times it was less so." This is the reason why researchers are taught to follow rigorous methods to guard against their own biases during such decision-making processes; that is what makes it SCIENCE!

Rigorous methods would have given group members just the methods sections of all studies meeting their selection criteria with author, title and journal names omitted. Then, each group member would independently make the control group, "most proximal," and "probably 
blinded" assignments with inter-rater reliabilities calculated and reported for each of their 12 meta-analyses including a similarly blinded method for resolving disagreements in adherence with their prespecified protocols. Such practices form the basics of scientific methodology and are necessary to protect Sonuga-Barke et al.'s 12 meta-analyses from potential bias either for or against specific treatments by blinding study outcomes when making assignments.

Our email to Sergeant asked specifically for evidence that the group had followed a method to ensure the internal reliability of their meta-analyses by keeping members blind to study outcomes when making assignments. While not answering our question directly, Sergeant's email response states they followed a consensus approach to these decisions with group members using "ALL AVAILABLE INFORMATION within the papers to make a judgment," apparently, even when these "consensus judgments" trumped their clearly stated protocols.

It is disappointing that a group of 21 researchers participated in such a flawed methodology with apparently none insisting on scientific rigor to protect their meta-analyses from researcher bias either for or against particular treatments. Instead, with full knowledge of the Steiner et al. results, out of the FOUR possible comparisons for their "probably blinded assessment" (i.e., parent NFB rating versus CT or waitlist; teacher NFB rating versus CT or waitlist), the researchers picked the ONLY comparison negative to NFB and in the process violated their TWO clearly stated protocols for guiding this decision (see Table 1).

These two protocol violations tipped the group's overall analysis to where NFB narrowly missed reaching significance in their "probably blinded" meta-analysis versus being found efficacious as NFB would have been if any of the other three possible comparisons had been used. The researchers then concluded that NFB requires "better evidence for efficacy from blinded assessments" before it can be supported as a treatment for ADHD. This is not science; it is researcher bias and incompetence masquerading as science.

Fundamentally, science is sabotaged when sound methodology is not followed as occurred in this group's meta-analyses as the 21 Sonuga-Barke et al. authors took no apparent efforts to protect against the biases of group members in the study's conduct, they included lowquality studies without assessing for their differential impact, and they failed to follow their own published protocols. These significant errors consign this group's study to the growing library of other meta-analytic studies exemplary of the "garbage-in-garbageout" phenomenon of poorly conducted meta-analyses that publish findings with sweeping conclusions unconstrained by their substandard methods.

\section{Steiner et al.'s Full Trial Results}

The NFB results from Steiner et al.'s subsequent full trial $(N=104)$ are particularly impressive (Steiner et al., 2014a). The full trial found NFB resulted in superior improvements in ADHD's core symptoms compared to both the CT and waitlist groups in executive functioning, parent ratings, teacher ratings, and blinded classroom observations as well as being the only group in which there were not significant increases in stimulant medication dosing during the study (Steiner et al., 2014a). Finally, the clear superiority of NFB over both the CT and waitlist conditions was then sustained in the 6-month follow-up assessment including on the medication dosing measure (Steiner et al., 2014b). 
Given the group's protocol violations in how they evaluated the Steiner et al. preliminary study, other significant methodological errors, and, more importantly, the results from Steiner's full trial with 6-month follow-up, the weight of the evidence overwhelmingly favors Arns et al.'s (2014) conclusion that standardized NFB treatment protocols have demonstrated efficacy and, at minimum, a medium effect size for ADHD's core symptoms in randomized trials using semi-active and active control groups.

\section{Who is the European ADHD Guidelines Group?}

It is worth noting that the self-named "European ADHD Guidelines Group" is a workgroup of the European Network for Hyperkinetic Disorders (ENHD), and the guideline group's effort was funded by five pharmaceutical companies. Neither entity has any apparent official "European" status other than being made up of European researchers, many of whom have extensive conflicts of interests (COls) with pharmaceutical companies. For example, the Sonuga-Barke et al. authors had a cumulative total of 114 reported COls with pharmaceutical companies in their disclosure statement.

It is unfortunate that this group of researchers with such extensive pharmaceutical COls decided to conduct 12 meta-analyses on non-pharmacological treatments for ADHD without ensuring rigorous methodological controls of their own biases. These biases were blatant in their handling of the Steiner et al. preliminary study and are likely present elsewhere in their other analyses if carefully reviewed. Our view is that the extent of scientific error warrants the article being retracted in its current form. It is even more unfortunate that this article was published in the American Journal of Psychiatry, a high impact journal, and has been widely cited as finding that four non-pharmacological treatments require "better evidence for efficacy from blinded assessments . . . before they can be supported as treatments for core ADHD symptoms," a finding that is clearly not true for NFB and perhaps other non-pharmacological treatments as well.

\section{Evidentiary Bias}

Similar to Barkley, this network of researchers demonstrates significant evidentiary bias in how they assess the evidence-base of their preferred treatments for ADHD and neurofeedback. The ENHD group's website recommends psycho-education, parent training, school-based interventions and cognitive behavioral training as "evidence-based" treatments-recommendations DIRECTLY counter to their OWN published metaanalyses in which these behavioral interventions performed the WORST out of the SIX non-pharmacological treatments they analyzed - while it lists NFB as an "area of controversy in the treatment of ADHD" and goes on to state that "more high quality, randomized controlled studies are needed to support neurofeedback training as a treatment for ADHD" (ENHD website).

It is unbecoming for a group of researchers to convey such false information to the public as to the relative evidence-base for different treatments. What's good for the goose is good for the gander. Evidentiary standards should be evenly applied as is clearly not the case on the ENHD's website.

Perhaps it is only coincidental, but several of the more prominent members of the European research network receive royalties from behavioral intervention products for treating ADHD. For example, the conflict of interest section at the beginning of the article states, "Dr. Sonuga-Barke has been involved in the development, implementation, and trialing of the 
New Forest Parenting Programme for preschool children with ADHD and has received royalties from sales of a New Forest Parent Training self-help manual" (Sonuga-Barke et al., 2013). Could it be that such conflicts of interest help explain why the network's website is so tolerant of evidentiary bias that favors their own group members' commercial interests but is directly counter to their own published findings?

\section{Negative Findings in Recent Sham Trials}

Three recent studies failed to find significant separation between "real" NFB and shamfeedback, raising doubts about the specificity of NFB effects. Two studies used nonstandardized protocols and training procedures contrary to operant conditioning (see Arns et al., 2014 for detailed review) while the third randomized only 9 subjects, and the authors, therefore, did not evaluate for specific effects (Perreau-Linck et al., 2010).

The first study was an NIMH-funded feasibility trial that was explicitly designed NOT TO BE an efficacy study due to its small sample size $(N=39)$ and the study design randomizing treatment frequency. For blinding purposes, this study attempted to train decreased theta/alpha and increased SMR/beta using an automated procedure in which the EEG threshold necessary to play SonyPlayStation ${ }^{\circledR}$ videogames was reset every minute (up or DOWN) based on subjects' immediately preceding EEG. The auto-threshold was set to ensure NFB subjects played videogames with full-control approximately 75 to $80 \%$ of the time during their sessions. When this NFB strategy failed to separate from sham-feedback, the authors noted that this may have been due to their inadequate NFB training protocol stating, "In fact, many NF experts feel that manually adjusted thresholds that remain fixed for periods of time work better than the fuzzy-logic moment-to-moment adjustments used in the CyberLearning technology used by us. Therefore, we do not have as much confidence in $\mathbf{3 0}$ treatments showing the maximal (NFB) effect' [emphasis added; Arnold et al., 2013b].

\section{The Lansbergen/van Dongen-Boomsma/Vollebregt Trilogy}

The second trial involved an initial pilot study using similar auto-thresholding procedures as used by Arnold et al. (Lansbergen et al., 2011). The children assigned to NFB watched a movie 20 minutes per session for 30 sessions. Positive feedback was provided by both brightening the computer screen and presenting an auditory tone when the targeted brainwave frequencies remained above/below the targeted thresholds using a computerized auto-thresholding procedure that reset the threshold every " 30 seconds so that the child was rewarded about $80 \%$ of the time (i.e., received positive feedback)" and "the amount of reward remained at about the same level across sessions and across groups. During training, children were instructed to try to self-regulate their brain activity by receiving positive feedback based on the real-time EEG signal ... Training was conducted in an 'active focusing state' with eyes open" (Lansbergen et al., 2011).

When Lansbergen et al.'s NFB training protocol failed to separate from their sham feedback condition for the 14 pilot study subjects, the authors hypothesized that it might be due to their use of auto-thresholding and decided to instead have a therapist manually perform the same re-thresholding function for the additional 14 subjects randomized to NFB. In the first published article with these additional 14 NFB subjects, the authors state, "Reward thresholds were manually adjusted so that the child was rewarded about $80 \%$ of the time (i.e., received positive feedback). Consequently, the amount of reward remained about at the same level across sessions and across groups" [emphases added; van 
Dongen-Boomsma et al., 2013]. It is important to note that this is the same "about $\mathbf{8 0 \% "}$ reward criteria maintained "at the same level across sessions and across groups" as in the pilot study's NFB training protocol except now NFB therapists were manually implementing it.

\section{Two-Thirds Underpowered, Mid-Stream Changes and Selective Reporting}

Despite changing their research design mid-stream (including adding undefined "active learning strategies ... so that children could apply the learned strategies into daily life") and being two-thirds underpowered having randomized only 41 of the 120 intended subjects their own analysis had determined was necessary prior to starting the study; the authors pooled their underpowered dataset and published two additional articles finding no specific NFB effects on behavioral and neurocognitive outcomes. The authors then claimed, "The existing literature and this study fail to support any benefit of neurofeedback" [emphasis added; Vollebregt et al., 2013). The Arnold and Perreau-Linck trials, along with the European guideline group's article, were a significant part of the "existing literature" used to support the authors' claim.

In their discussion section, Vollebregt et al. state, "The most likely explanation why we did not find improvement of neurocognitive functioning after F-NF is that F-NF is not an effective treatment in ADHD. This conclusion is in line with THREE recently published placebo-controlled F-NF studies reporting no superior effect on the core behavior symptoms of ADHD (Arnold et al., 2012; van Dongen-Boomsma et al., 2013; Lansbergen et al., 2011; Perreau-Linck et al., 2010)" [emphases added; Vollebregt et al., 2013]. Vollebregt et al. fail to acknowledge in this highly critical and misleading statement that:

- Two of the four referenced studies were part of their own trial, and by the authors' own analysis, their study was two-thirds underpowered; to form even this underpowered dataset, the researchers pooled subjects after making two significant changes in their research design mid-way through the study;

- The Arnold et al. trial was explicitly a feasibility study, not an efficacy trial, and its authors specifically stated they did not have confidence in their NFB's results due to their study's minute-by-minute re-thresholding of the EEG reward criteria, contrary to the recommendations of, as they state, the "NF experts"; and

- The Perreau-Linck et al. study had only 9 subjects (7 completers), and the authors clearly state that "the small sample size precludes from evaluating specific neurofeedback effects" [emphasis added; Perreau-Linck et al., 2010].

Certainly, these facts from the referenced articles by others should have been acknowledged by Vollebregt et al. so that journal readers could then judge for themselves the extent to which the "existing research" supports the authors' claims.

Whose Neurofeedback Training Protocol did Lansbergen/van Dongen-Boomsma/Vollebregt Follow?

Another highly misleading assertion by Vollebregt et al. is when they state, "Thresholds were manually adjusted according to the expertise of the NF-therapist. NO specific guideline or protocol was followed. This method was in line with the OBJECTIVE OF THIS STUDY to investigate the efficacy of F-NF as delivered in 'CARE AS USUAL', in which decisions about adjustments of the threshold are determined by the involved clinical 
NF-therapist. All of the NF-therapists were BCIA certified (Board Certification International Alliance)" [emphases added].

As made clear in van Dongen-Boomsma et al., instead of the automatically adjusted thresholds, the NFB therapists were to manually adjust them such that "the child was rewarded about $80 \%$ of the time." This decision was the RESEARCHERS' PROTOCOL ADAPTATION to maintain as best they could the same "about $80 \%$ " level of reward "across sessions and across groups" as they had in the pilot study with the NFB therapists using their software skills to continuously monitor each subjects' EEG training targets and then to the best of their ability adjust up or down the reward thresholds for each target so that "the child was rewarded about $80 \%$ of the time... across sessions." The NFB "therapists" were merely attempting to follow the RESEARCHERS" "about 80\%" reward guideline/protocol. The NFB "therapists" did not use their NFB expertise to treat these children's ADHD; they used their knowledge of the NFB software to implement the researchers' protocol to the best of their ability, nothing more.

Vollebregt et al. add to their lack of scientific transparency by then claiming, "This method was in line with the OBJECTIVE OF THIS STUDY to investigate the efficacy of F-NF as delivered in 'CARE AS USUAL', in which decisions about adjustments of the threshold are determined by the involved clinical NF-therapist." There is no evidence in the prior Lansbergen/van Dongen-Boomsma et al. articles for this claim on Vollebregt et al.'s part.

This was certainly NOT NFB "CARE AS USUAL" treatment for ADHD or any other disorder. Yes, the NFB "therapists" utilized their NFB software expertise to implement to the best of their ability the researchers' "about $80 \%$ " reward guideline/protocol; but it was the researchers' protocol (including how the EEG training targets were selected in the first place) that the "therapists" were attempting to implement.

Vollebregt et al. then add further to the confusing and misleading presentation of their methods by stating, "All of the NF-therapists were BCIA certified" as though this adds legitimacy to their claim that this was a study of NFB "CARE AS USUAL," now with the added moniker, "as provided by BCIA certified therapists." We do not doubt that the "therapists" were BCIA certified, but their BCIA training, certification, and NFB competence were not used in this study, only their expertise in using the NFB software in their attempts to adhere to the researchers' $80 \%$ reward protocol; NOTHING MORE!

Vollebregt et al.'s disingenuous presentation misleads journal readers and the public-at-large into believing that theirs was a study of NFB "CARE AS USUAL" for ADHD as provided by "BCIA certified" therapists and that their findings failed "to support any benefit of neurofeedback." Vollebregt et al. then repeat this false "CARE AS USUAL" claim three more times in their discussion section, thereby tarnishing further the BCIA certification accomplishment of those NFB therapists achieving this designation, discouraging other professionals from seeking BCIA Neurofeedback Board Certification, harming NFB therapists' professional reputation and ability to earn a living from their chosen profession, and most importantly, harming ADHD children and their families by discouraging them from seeking the best currently available first-line treatment for ADHD (Pigott \& Cannon, 2014).

\section{What "Active Learning Strategies" did the Researchers Use?}

As stated previously, the van Dongen-Boomsma/Vollebregt et al. researchers added "active learning strategies" mid-stream during their study "so that children could apply the learned strategies into daily life," without describing what these "learning strategies" were. 
Furthermore, they did not state what they did to ensure that these strategies were in fact learned; otherwise, how could the children then apply them outside the treatment setting "into daily life"?

It is only by reading the Lansbergen et al. pilot study's discussion section that we learn that "a third limitation of this study is the lack of actively practicing mental strategies to selfregulate brain activity (e.g., Gevensleben et al., 2009). In other words, EEG-neurofeedback might need explicit learning rather than implicit learning . . . Based on these results ... we will assist children in developing and practicing active learning strategies to selfregulate brain activity (e.g., focusing one's attention) and promote the children to implement the acquired strategies in daily-life situations, aimed at optimizing the therapeutic effects of EEG-neurofeedback training (see Gevensleben et al., 2009)" [emphases added; Lansbergen et al., 2011].

We reviewed Gevensleben et al. to ascertain what "active learning strategies" van DongenBoomsma/Vollebregt et al. are referencing that make NFB learning "explicit" rather than "implicit." Gevensleben et al. state, "Transfer trials, i.e., trials without contingent feedback, were also conducted (about $40 \%$ at the beginning of a training block and about $60 \%$ at the end of a training block). The children of the NF group were required to practice their focused state (which was practiced in the sessions) at home, in different situations (one situation per day, e.g., 'try to be very focused while reading', 'try stay focused on the ball while playing football this afternoon')." Gevensleben et al.'s use of transfer trials and home practice is the same as that used in Gani et al.'s (2008) two-year follow-up study concluding that, "Neurofeedback appears to be an alternative or complement to traditional treatments. The stability of changes might be explained by normalizing of brain functions that are responsible for inhibitory control, impulsivity and hyperactivity." Gevensleben et al. (2010) found a similar maintenance of NFB treatment gains in their 6-month follow-up assessment.

So again we ask, "What 'active learning' methodology did van Dongen-Boomsma/Vollebregt use?" They present no evidence of implementing Gevensleben et al.'s transfer trial strategies that do, in fact, make NFB learning "explicit" rather than "implicit." This second mid-stream adaptation by van Dongen-Boomsma/Vollebregt et al. is not only a confounding variable in their study, but by leaving it undefined, the authors tarnish the value of these researchers' thoughtful approach as well as the continued research efforts on how best to generalize NFB learning in the laboratory to everyday life situations.

\section{News Alert: $A D H D$ Children are as Smart as Hungry Pigeons}

While the Lansbergen/van Dongen-Boomsma/Vollebregt trilogy acknowledges that NFB "is based on the rationale that voluntary modulation of specific brain activity patterns can be learned by operant learning strategies" (Vollebregt et al., 2013), their methodology fails basic principles of operant conditioning. As behaviorists know, learning will not occur if the reinforcement criteria are made more or less stringent in order to maintain a high frequency of reward (e.g., Thorndike, 1932). The issue is not auto-thresholding versus "therapists" performing the same function, rather it is the failure to shape the EEG. For example, if hungry pigeons are fed a pellet approximately 8 out of 10 times they peck with the accuracy criteria made more or less stringent to maintain this high frequency of reward for 30,40 , or $100+$ sessions, they will be no more accurate in pecking the target when they finish "treatment" than when they started, since pecking accuracy was not shaped. 
Similarly, NFB subjects' EEGs were not reliably shaped, since regardless of their success at producing the desired EEG, they watched movies/played SonyPlayStation $\circledast$ videogames without disruption approximately 70 to $80 \%$ of the time. Furthermore, watching movies/playing videogames was likely far more reinforcing than striving to control the intermittent disruptions, particularly since most NFB subjects would soon learn that they could not control these disruptions on a sustained basis anyway, as their EEG thresholds were always being reset according to the authors' "about $80 \%$ " reward protocol regardless of how hard they tried . . . so why bother? The smart strategy for all subjects, therefore, was to simply ignore these minor disruptions, and instead, enjoy the entertainment similar to hungry pigeons happy to be fed for aimless pecking.

This "kick back and enjoy the show mindset" is what occurred, since in a subsequent article, the authors state, "Most participants of EEG-NF placebo-controlled RCTs conducted until now seem to experience the treatment as a placebo condition" and then cite all three articles from their trilogy along with one other (Vollebregt et al., 2014).

Given both the Arnold et al. and Lansbergen/van Dongen-Boomsma/Vollebregt et al. studies' flawed NFB training methodology, it is not surprising that their blinds were not broken and no evidence of specific effects found since both studies essentially compared sham treatments. Arnold et al.'s failure to shape NFB subjects' EEGs is made clear when they reported that "the sham group (AS WELL AS ACTIVE GROUP) showed no obvious EEG changes in a simple pre-post measure of theta/beta ratio" [emphasis added; Arnold et al., 2013a].

Furthermore, Vollebregt et al. found far more evidence of the NEGATIVE SHAPING of NFB subjects' EEGs away from their training targets than shaping in the desired direction. Their results section states, "EEG-data during the sessions were available for 10 children . . .

Seven children showed a change in power towards ONE of the training targets. However, the variability between sessions was great and no children showed such a desired change in more than ONE frequency-band. Moreover, ALL CHILDREN additionally showed a change in power AWAY FROM A TRAINING TARGET' [emphases added; Vollebregt et al., 2013].

In the table of the EEG training targets for all 22 NFB subjects, 14 had two EEG training targets and 8 had three targets for an average of 2.4 EEG training targets per NFB subject (supplemental Table 2; van Dongen-Boomsma et al., 2013). While the authors report that there was EEG data available for only 10 NFB subjects, assuming that these subjects were representative of the NFB group as whole, there were approximately 24 EEG training targets on which data was available to evaluate the ability of their training methodology to shape subjects' EEG. Out of approximately 24 EEG training targets, Vollebregt et al. report a change in power in the desired direction in only seven (29\%), with a minimum of ten changes in power in the negative direction and possibly 17 or more instances of the NEGATIVE SHAPING of NFB subjects' EEG (range: 42 to $71+\%)$.

Therefore, despite the researchers instructing the NFB subjects "to attempt to self-regulate their brain activity by receiving positive feedback" and given that the "reward thresholds [were] manually adjusted so that the child was rewarded about $80 \%$ of the time" (van Dongen-Boomsma et al., 2013), the ADHD children proved to be as smart as pigeons. The NFB subjects quickly learned that any of their attempts to "self-regulate their brain activity by receiving positive feedback" were futile because the reward criteria was intermittently being changed to maintain the researchers' "about $80 \%$ " positive feedback protocol. So like hungry pigeons happy to be fed for aimless pecking, the NFB subjects soon learned to just kick back and enjoy the show. 
This study's findings are as expected, since how can learning take place when the accuracy criteria necessary to receive "positive feedback" is continually changed to maintain a reward frequency of "about $80 \%$ " even when the participants are getting worse in performing the behavior to be learned? The Lansbergen/van Dongen-Boomsma/Vollebregt trilogy proves that it cannot, as did Thorndike, Skinner and many other behaviorists long before. Bad science confirms the good; how fitting.

\section{Subterfuge versus Acknowledging the Evidence}

In their discussion section, Vollebregt et al. fail to acknowledge the evidence indicating that their training methodology negatively shaped NFB subjects' EEGs away from their training targets and then offered this fact as the most likely reason that their NFB protocol failed to separate from sham feedback. If, as Vollebregt et al. state, NFB "is based on the rationale that voluntary modulation of specific brain activity patterns can be learned by operant learning strategies," certainly evidence demonstrating the negative shaping of NFB subjects' EEGs warrants discussion by the authors. Instead of acknowledging this evidence, Vollebregt et al. only state in their discussion section that "NOT ALL desired training directions were met" when they should have been forced by competent peer and editorial review to acknowledge the fact that there was far more evidence of the negative shaping of NFB subjects' EEGs away from their training targets than shaping in the desired direction. Such an acknowledgement is the minimum standard of researcher objectivity when discussing study findings.

\section{Methodology Matters}

Concerned about the proliferation of unsound practices, many leading researchers published a consensus statement on NFB and basic learning theory (Sherlin et al., 2011). Key points included:

- How readjusting the EEG threshold up and down during NFB sessions to maintain a high frequency of reward violates the principal of shaping and may in fact shape it, "in the opposite direction than the desired training parameter" as occurred in Lansbergen/van Dongen-Boomsma/Vollebregt et al.;

- Monetary or other secondary reinforcers should be based on success shaping the EEG, NOT mere participation in NFB;

- Encouraging strategies to promote generalization such as brief transfer trials where trainees attempt to activate the targeted cortical activity via self-regulation alone without real-time feedback and only learn if they were successful at the end of the trial; and

- Sessions should "stress exercise rather than entertainment" with the reinforcement leading to "knowledge of results" and informing "the learner whether the response was right or wrong and to what extent the brain signal changed."

While exemplifying unsound methodologies (including giving reinforcers for participation, not performance), these failed trials significantly advance scientifically-based NFB treatment by demonstrating that flawed practices fail to shape trainees' EEGs and consequently render outcomes indistinguishable from sham feedback. Using similarly flawed methodologies (e.g., re-thresholding to ensure subjects watched movies disruption-free 70 to $85 \%$ of the time and giving reinforcers for participation, not performance), Ogrim and Hestad (2013) also found such practices failed to shape trainees' EEGs. 
The negative findings from these studies are in marked contrast to those from Leins et al. (2007) whose methods closely followed those in the consensus statement including the use of transfer trials and providing secondary reinforcers based on subjects' performance learning to self-regulate their EEG. This study compared theta/beta ratio and slow cortical potential training and found that both groups (a) learned to self-regulate cortical activity consistent with their NFB training protocol; (b) had significant improvements in the core symptoms of ADHD as well as IQ; (c) EEG self-regulation was maintained when reassessed six months and two years later; (d) a two-year follow-up found further significant improvements in behavior and attention; and (e) in each group, half of the children no longer met the criteria for ADHD (Gani, Birbaumer, \& Strehl, 2008). Other evidence of NFB's specificity are studies finding significant neurophysiological effects including protocol-specific effects on event-related potential (ERP) components in attention tasks and neural substrates of selective attention imaged with fMRI (see Arns et al., 2014). In addition to Leins and Steiner finding strong evidence of sustained improvement in follow-up assessments of methodologically sound NFB procedures, every other NFB study that has included follow-up assessments has found similar evidence of sustained improvements in protocol-specified EEG and ADHD core symptoms. No other ADHD treatment has demonstrated credible evidence of sustained benefit following treatment termination; NONE (Pigott \& Cannon, 2014).

\section{Conclusion}

It is our assessment that the critics' claims that neurofeedback lacks sufficient evidence of efficacy and, therefore, cannot be considered a legitimate treatment for ADHD do not stand up to careful scrutiny. The critics making these claims display significant evidentiary bias in how they evaluate neurofeedback compared to treatments in which they have commercial interests (e.g., Barkley's website; ENHD's website), and the scientific rigor of methods supporting their conclusions are questionable.

The Sonuga-Barke et al. and Vollebregt et al. articles bring into question the scientific rigor adhered to during these studies' peer review and publication process. Sandra Loo, Russell Barkley, and the authors of the other two studies have all been invited to give a formal response to the methodological issues we have raised. Our view is that $A L L$ authors should either defend the scientific integrity of the claims to which their names are assigned or formally request that their articles be retracted. Anything less is an abdication of scientific duty on their part and a de facto acknowledgement that they have no credible response.

In contrast to these critics' claims, the actual evidence is that neurofeedback, when administered in a way that is consistent with the principles of operant conditioning and using standardized treatment protocols (Arns et al., 2014), is the best currently available first-line treatment for ADHD (Pigott \& Cannon, 2014). With full acknowledgement of our own commercial interests as neurofeedback professionals, this is a claim we will vigorously defend and invite critics of neurofeedback to challenge. 


\section{References}

Arnold, L. E., Arns, M., Conners, K., deBeus, R., Hirshberg, L., \& Monastra, V. (2013a). A proposed multisite double-blind randomized clinical trial of neurofeedback for ADHD: Need, rationale, and strategy. Journal of Attention Disorders, 17, 420-436. http://dx.doi.org/10.1177/1087054713482580

Arnold, L. E., Lofthouse, N., Hersch, S., Pan, X., Hurt, E., Bates, B . . Grantier, C. (2013b). EEG neurofeedback for ADHD: Double-blind sham-controlled randomized pilot feasibility trial. Journal of Attention Disorders, 17(5), 410-9. http://dx.doi.org/10.1177/1087054712446173

Arns, M., Heinrich, H., \& Strehl, U. (2014). Evaluation of neurofeedback in ADHD: The long and winding road. Biological Psychology, 95, 108-115.

http://dx.doi.org/10.1016/j.biopsycho.2013.11.013

Arns, M., \& Strehl, U. (2013). Evidence for efficacy of neurofeedback in ADHD? (letter). American Journal of Psychiatry, 170, 799. http://dx.doi.org/10.1176/appi.ajp.2013.13020208

Bakhshayesh, A. R., Hansch, S., Wyschkon, A., Rezai, M. J., \& Esser, G. (2011). Neurofeedback in ADHD: A single-blind randomized controlled trial. European Child \& Adolescent Psychiatry, 20, 481-491. http://dx.doi.org/10.1007/s00787-011-0208-y

Barkley, R. A. (1987). Defiant children: A clinician's manual for parent training. New York, NY: Guilford Press.

Barkley, R. A. (1992). Is EEG biofeedback treatment effective for ADHD children? Proceed with much caution. Attention Deficit Disorder Advocacy Group newsletter.

Barkley, R. website accessed April 29, 2014: http://www.russellbarkley.org/factsheets/adhdfacts.pdf.

Cannon, R. L. (in press). Parietal foci for Attention Deficit/Hyperactivity Disorder: Targets for LORETA neurofeedback with outcomes. Biofeedback Magazine: Special issue on QEEG and Treatment Approaches for ADHD, June 2014.

Cannon, R. L., Baldwin, D., Diloreto, D., Phillips, S., Shaw, T. L., Levy, J. J. (2014). LORETA neurofeedback in the precuneus: Operant conditioning in basic mechanisms of selfregulation. Clinical EEG and Neuroscience, DOI: 10.1177/1550059413512796. http://dx.doi.org/10.1177/1550059413512796

ENHD website accessed April 29, 2014: http://adhd-adduce.org/page/view/55/What+nondrug+treatments+help

FDA website accessed April 29, 2014: http://www.accessdata.fda.gov/drugsatfda docs/label/2003/21475 methylin lbl.pdf.

Gani, C., Birbaumer, N., \& Strehl, U. (2008). Long term effects after feedback of slow cortical potentials and of theta-beta-amplitudes in children with attention-deficit/hyperactivity disorder (ADHD). International Journal of Bioelectromagnetism, 10(4), 209-232. 
Gevensleben, H., Holl, B., Albrecht, B., Vogel, C., Schlamp, D., Kratz, O., ... Heinrich, H. (2009). Is neurofeedback an efficacious treatment for ADHD? A randomized controlled clinical trial. Journal of Child Psychology and Psychiatry, 50(7), 780-789. http://dx.doi.org/10.1111/j.1469-7610.2008.02033.x

Gevensleben, H., Holl, B., Albrecht, B., Schlamp, D., Kratz, O., Studer, P., \& Heinrich H. (2010). Neurofeedback training in children with ADHD: 6-Month follow-up of a randomized controlled trial. European Child \& Adolescent Psychiatry, 19, 715-724. http://dx.doi.org/10.1007/s00787-010-0109-5

Heinrich, H., Gevensleben, H., Freisleder, F. J., Moll, G. H., \& Rothenberger, A. (2004). Training of slow cortical potentials in attention-deficit/hyperactivity disorder: Evidence for positive behavioral and neurophysiological effects. Biological Psychiatry, 55(7), 772-775. http://dx.doi.org/10.1016/j.biopsych.2003.11.013

Holtmann, M., Grasmann, D., Cionek-Szpak, E., Hager, V., Panzer, N., Beyer, A., \& Stadler, C. (2009). Specific effects of neurofeedback on impulsivity in ADHD. Kindheit und Entwicklung, 18, 95-104. http://dx.doi.org/10.1026/0942-5403.18.2.95

Lansbergen, M. M., van Dongen-Boomsma, M., Buitelaar, J. K., \& Slaats-Willemse, D. (2011). ADHD and EEG-neurofeedback: A double-blind randomized placebocontrolled feasibility study. Journal of Neural Transmission, 118(2), 275-84. http://dx.doi.org/10.1007/s00702-010-0524-2

Leins, U., Goth, G., Hinterberger, T., Klinger, C., Rumpf, N., \& Strehl, U. (2007). Neurofeedback for children with ADHD: A comparison of SCP and Theta/Beta protocols. Applied Psychophysiology and Biofeedback, 32, 73-88. http://dx.doi.org/10.1007/s10484-007-9031-0

Levesque, J., Beauregard, M., \& Mensour, B. (2006). Effect of neurofeedback training on the neural substrates of selective attention in children with attention-deficit/hyperactivity disorder: A functional magnetic resonance imaging study. Neuroscience Letters, 394, 216-221. http://dx.doi.org/10.1016/j.neulet.2005.10.100

Molina, B. S., Hinshaw, S. P., Swanson, J. M., Arnold, L. E., Vitiello B., Jensen, P. S., . . MTA Cooperative Group. (2009). The MTA at 8 years: Prospective follow-up of children treated for combined-type ADHD in a multisite study. Journal of the American Academy of Child \& Adolescent Psychiatry, 48(5), 484-500. http://dx.doi.org/10.1097/chi.0b013e31819c23d0

Olfson, M., Blanco, C., Liu, S. M., Wang, S., \& Correll, C. U. (2012). National trends in the office-based treatment of children, adolescents, and adults with antipsychotics. Archives of General Psychiatry, 69(12), 1247-56. http://dx.doi.org/10.1001/archgenpsychiatry.2012.647

Ogrim,G., \& Hestad, K.A. (2013). Effects of neurofeedback versus stimulant medication in attention-deficit/hyperactivity disorder: A randomized pilot study. Journal of Child and Adolescent Psychopharmacology, 23(7), 448-457. http://dx.doi.org/10.1089/cap.2012.0090 
Perreau-Linck, E., Lessard, N., Levesque, J., \& Beauregard, M. (2010). Effects of neurofeedback training on inhibitory capacities in ADHD children: A single-blind, randomized, placebo-controlled study. Journal of Neurotherapy, 14, 229-242. http://dx.doi.org/10.1080/10874208.2010.501514

Riddle, M., Yershova, K., Lazzaretto, D., Paykina, N., Yenokyan, G., Greenhill, L., \& Posner, K. (2013). The preschool attention-deficit/hyperactivity disorder treatment study (PATS) 6-year follow-up. Journal of the American Academy of Child \& Adolescent Psychiatry, 52(3), 264-278. http://dx.doi.org/10.1016/j.jaac.2012.12.007

Sherlin, L. H., Arns, M., Lubar, J., Heinrich, H., Kerson, C., Strehl, U., \& Sterman, M. B. (2011). Neurofeedback and basic learning theory: Implications for research and practice. Journal of Neurotherapy, 15, 292-304. http://dx.doi.org/10.1080/10874208.2011.623089

Smith, G., Jongeling, B., Hartmann, P., Russell, C., \& Landu, L. (2010). Raine ADHD study: Long-term outcomes associated with stimulant medication in the treatment of ADHD in children. Department of Heath, Western Government of Australia.

Snyder, S. M., Quintana, H., Sexson, S. B., Knott, P., Haque, A. F., Reynolds, D. A. (2008). Blinded, multi-center validation of EEG and rating scales in identifying ADHD within a clinical sample. Psychiatry Research, 159, 346-358. http://dx.doi.org/10.1016/i.psychres.2007.05.006

Sonuga-Barke, E. J., Brandeis, D., Cortese, S., Daley, D., Ferrin, M., Holtmann, M., . . Sergeant, J. (2013a). Nonpharmacological interventions for ADHD: Systematic review and meta-analyses of randomized controlled trials of dietary and psychological treatments. American Journal of Psychiatry, 170(3), 275-289. http://dx.doi.org/10.1176/appi.ajp.2012.12070991

Sonuga-Barke, E. J., Brandeis, D., Cortese, S., Daley, D., Ferrin, M., Holtmann, M., . . Sergeant, J. (2013b). Response to Chronis-Tuscano et al. and Arns and Strehl (letter). American Journal of Psychiatry, 170, 800-802. http://dx.doi.org/10.1176/appi.ajp.2013.13020208r

Steiner, N. J., Sheldrick, R. C., Gotthelf, D., \& Perrin, E. C. (2011). Computer-based attention training in the schools for children with attention deficit/hyperactivity disorder: A preliminary trial. Clinical Pediatrics, 50, 615-622. http://dx.doi.org/10.1177/0009922810397887

Steiner, N. J., Frenette, E. C., Rene, K. M., Brennan, R. T., \& Perrin, E. C. (2014a). Neurofeedback and cognitive attention training for children with attention-deficit hyperactivity disorder in schools. Journal of Developmental \& Behavioral Pediatrics, 35, 18-27. http://dx.doi.org/10.1097/dbp.0000000000000009

Steiner, N. J., Frenette, E. C., Rene, K. M., Brennan, R. T., \& Perrin, E. C. (2014b). In-school neurofeedback training for ADHD: Sustained improvements from a randomized control trial. Pediatrics, 133, 483-492. http://dx.doi.org/10.1542/peds.2013-2059d

Thorndike, E. L. (1932). Reward and punishment in animal learning. Comparative Psychology Monographs, 8(39), 1-23. 
van Dongen-Boomsma, M., Vollebregt, M. A., Slaats-Willemse, D., Buitelaar, J. K. (2013). A randomized placebo-controlled trial of electroencephalographic (EEG) neurofeedback in children with attention deficit/hyperactivity disorder. Journal of Clinical Psychiatry, 74(8), 821-827. http://dx.doi.org/10.4088/jcp.12m08321

Vollebregt, M. A., van Dongen-Boomsma, M., Buitelaar, J. K., \& Slaats-Willemse, D. (2013). Does EEG-neurofeedback improve neurocognitive functioning in children with attention-deficit/hyperactivity disorder? A systematic review and a double-blind placebo-controlled study. Journal of Child Psychology and Psychiatry, 55(5), 460472. http://dx.doi.org/10.1111/jepp.12143

Vollebregt, M. A., van Dongen-Boomsma, M., Slaats-Willemse, D. \& Buitelaar, J. K. (2014). What future research should bring to help resolving the debate about the efficacy of EEG-neurofeedback in children with ADHD. Frontiers in Human Neuroscience, 8, 321. http://dx.doi.org/10.3389/fnhum.2014.00321 


\section{Integrated Neuroscience Services}

provides individualized, patient-centered data analysis and consultations to clinicians. At INS, neuroscientific, neuropsychological and neurophysiological information is incorporated to facilitate the clinician's understanding of their patients' conditions, ultimately allowing the clinician to provide superior treatment. Specifically, we analyze and interpret EEG, QEEG, neuropsychological or other neurophysiological data. This analysis is discussed during the online video consultation, along with customized treatment recommendations.

\section{services}

$>$ Complete QEEG Analysis

$>$ Coherence Analysis $>$ Spike/Seizure Analysis

$>$ EEG Interpretation by Neurologist and Complex Medical-Legal Reports

$>$ Neuropsychological Data Review

$>$ Online Consultations

$>$ Online Supervision

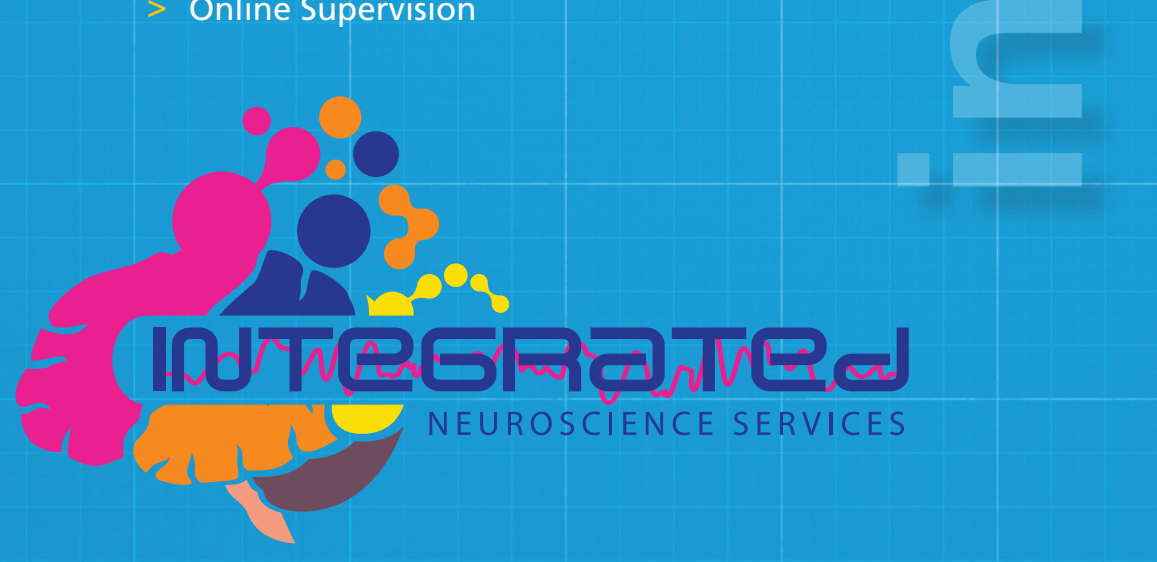

\section{company bio}

Integrated Neuroscience Services is the only company founded and operated by two clinical neuropsychologists. Dr. Coben and Dr. Stevens provide neurofeedback services daily and have directly helped over 20,000 of their own patients, in a combined 40 years of clinical practice. As such, their unique expertise is in EEG and QEEG data processing, clinical competency, neuropsychology, and how to assemble this wealth of information to maximize treatment benefits.

Recently, our company formed a joint collaboration with Q-Metrx, Inc. to provide comprehensive Quantitative EEG Reporting and Consultation. We will continue to provide state-of-the-art QEEG analyses with an emphasis on advanced connectivity analyses and clinical consultation and mentoring. On-going consultation is offered to closely track progress of individual clients and make necessary changes to optimize training outcome.

Q-Metrx, Inc., directed by Jack Johnstone, Ph.D, will provide interpretation of EEGs by Board Certified Neurologists as well as full medical review of comprehensive QEEG reports. These services are intended to be used for complex medical cases and in situations where medical-legal documentation is needed.

All services can be accessed by uploading EEG data to servers at Integrated Neuroscience Services.

For additional information see: www.integratedneuroscienceservices.com, www.q-metrx.com or call (866) 936-1882. 


\section{Join ISNR Today!}

\section{Together We Make a Powerful Team}

Open the door to the valuable discounts, education, and networking opportunities available exclusively to ISNR members.

\section{Valuable Discounts:}

- Discounts on Annual Conference registration, ISNR publications, webinars, DVD's and more

- FREE advertising through your online profile on ISNR web site

Networking Opportunities:

- Countless networking opportunities at ISNR Conferences

- Exclusive access to colleagues in the field through ISNR's online Member Directory

\section{Education:}

- FREE subscriptions to NeuroRegulation and NeuroConnections

- Continuing Education training for enhancing your skills and knowledge

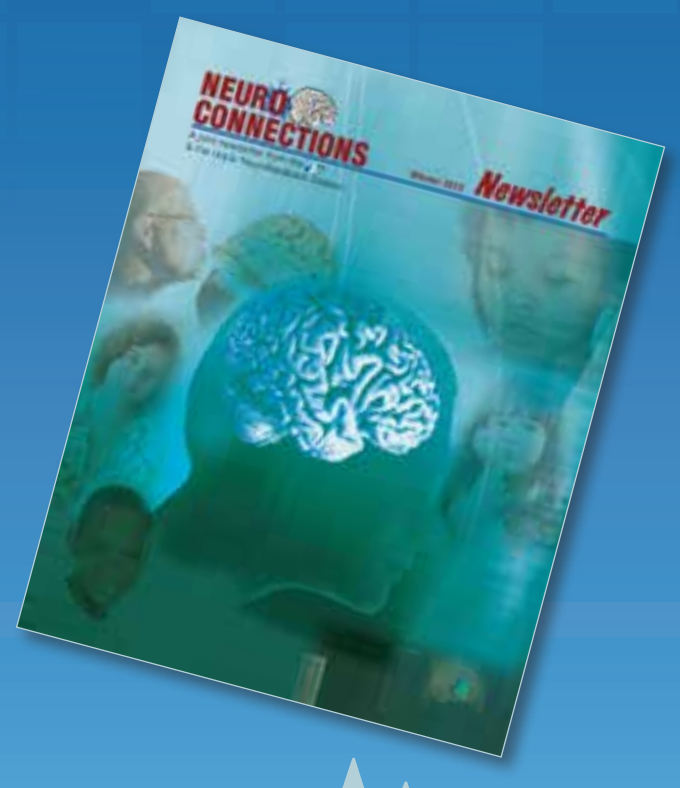

www.isnr.org 